\title{
Discriminative ability of reflex receptive fields to distinguish patients with acute and chronic low back pain
}

Monika Müller ${ }^{a, b}$, José A. Biurrun Manresa ${ }^{c}$, Fabienne Treichel ${ }^{a}$, Christoph A. Agten ${ }^{d}$, Paul Heini ${ }^{e}$, Ole K. Andersen ${ }^{c}$, Michele Curatolo ${ }^{\mathrm{c}, \mathrm{f}, \star}$, Peter Jüni ${ }^{\mathrm{g}, \mathrm{h}}$

\begin{abstract}
Low back pain has a life time prevalence of $70 \%$ to $85 \%$. Approximately $10 \%$ to $20 \%$ of all patients experience recurrent episodes or develop chronic low back pain. Sociodemographic, clinical, and psychological characteristics explain the transition from acute to chronic low back pain only to a limited extent. Altered central pain processing may be a contributing mechanism. The measurement of reflex receptive fields (RRF) is a novel method to assess altered central pain processing. The RRF area denotes the area of the foot sole from which spinal nociceptive reflexes can be elicited. It was shown to be enlarged in patients with acute and chronic low back pain compared with pain-free individuals. The aim of the study was to explore the discriminative ability of the RRF to distinguish patients with acute and chronic low back pain with the hypothesis that enlarged RRF are associated with chronic low back pain. We included 214 patients with either acute or chronic low back pain and compared RRF between groups in both univariable and multivariable analyses adjusted for different sociodemographic and clinical characteristics possibly associated with the transition to chronic pain. We found a mean difference between patients with acute and chronic low back pain of -0.01 ( $95 \%$ confidence interval [Cl], -0.06 to 0.04$)$ in the crude, $-0.02(95 \% \mathrm{Cl},-0.08$ to 0.04$)$ in the age and sex adjusted, and $-0.02(95 \% \mathrm{Cl},-0.09$ to 0.05$)$ in the fully adjusted model. Our results suggest that the enlargement of RRF area may not be associated with the transition from acute to chronic low back pain.
\end{abstract}

Keywords: Pain sensitivity, Central sensitization, Quantitative sensory tests, Reflex receptive field, Nociceptive withdrawal reflex

\section{Introduction}

Low back pain poses a major health burden with $70 \%$ to $85 \%$ of all people complaining of back pain at some time in their life. ${ }^{4}$ The socioeconomic impact of chronic low back pain is comparable with depression, heart disease, diabetes, and cancer. ${ }^{16,17,32} \mathrm{~A}$ survey conducted in 2007 in Switzerland concluded that $47 \%$ of women and $39 \%$ of men had suffered from back problems in the preceding weeks. ${ }^{53}$ Approximately $10 \%$ to $20 \%$ of all patients

Sponsorships or competing interests that may be relevant to content are disclosed at the end of this article.

a University Clinic of Anesthesiology and Pain Medicine, Inselspital Bern, Bern, Switzerland, ${ }^{b}$ Institute of Social and Preventive Medicine, University of Bern, Bern, Switzerland, ${ }^{c}$ Department of Health Science and Technology, Center for Sensory-Motor Interaction, Aalborg University, Aalborg, Denmark, ${ }^{d}$ Department of Radiology, Balgrist University Hospital, Zürich, Switzerland, ${ }^{e}$ Department of Orthopedics, Private Clinic Sonnenhof, Bern, Switzerland, ${ }^{f}$ Department of Anesthesiology and Pain Medicine, University of Washington, Seattle, WA, USA, ${ }^{g}$ Institute of Primary Health Care, University of Bern, Bern, Switzerland, ${ }^{h}$ Applied Health Research Centre (AHRC), Li Ka Shing Knowledge Institute of St. Michael's Hospital, University of Toronto, Toronto, ON, Canada

*Corresponding author. Address: Department of Anesthesiology and Pain Medicine, University of Washington, 1959 NE Pacific St, Box 356540, Seattle, WA 98195-6540, USA. Tel.: +1-206 543 2568; fax: +1-206 543 2958. E-mail address: curatolo@uw.edu (M. Curatolo).

Supplemental digital content is available for this article. Direct URL citations appear in the printed text and are provided in the HTML and PDF versions of this article on the journal's Web site (www. painjournalonline.com).

PAIN 157 (2016) 2664-2671

(C) 2016 International Association for the Study of Pain

http://dx.doi.org/10.1097/j.pain.0000000000000683 experience recurrent episodes or a chronic course of the disease. ${ }^{4,10,22,24,25}$

Sociodemographic, psychological, and clinical characteristics are associated with transition to chronic low back pain. $8,13,14,20-24,28,47,48,51$ However, systematic reviews were inconclusive regarding the relative importance of these predictors. ${ }^{10,31,40}$ Therefore, research investigating other potentially contributing mechanisms is needed. The paradigm of central hyperexcitability is a promising model focusing on altered processing in the central nervous system. ${ }^{15,54,55}$ Studies using quantitative sensory testing found that patients with chronic musculoskeletal pain conditions including low back pain display signs of central hyperexcitability. 5,37,39,41,50 The assessment of altered central pain processing using quantitative sensory testing typically includes the measurement of pain intensity or pain thresholds after the application of painful stimuli. ${ }^{39,41}$ However, the perception of pain is subjective, which may cause unwanted variation in test results depending on various factors that are difficult to control during the assessment, such as catastrophizing, ${ }^{18,42,43,52}$ cognitive attention, ${ }^{7}$ and hormonal status. ${ }^{19}$

Novel methods based on more objective parameters would be desirable. Assessment of the spinal nociceptive withdrawal reflex (NWR) as involuntary response to nociceptive stimulation is an established approach to measure spinal nociceptive hyperexcitability. ${ }^{5,37,50}$ Basic research indicated a modular organization of the NWR. ${ }^{2,11,45,46}$ Each muscle has a well-defined cutaneous receptive field, the reflex receptive field (RRF). Nociceptive input applied to that region evokes a withdrawal reflex in the muscle, whereas stimulation outside the region has no effect. ${ }^{49}$ Based on these findings, we 
developed a method to measure RRF in humans. ${ }^{3,36,38}$ After validating the experimental technique in healthy humans, ${ }^{3,36}$ we performed 2 case-control studies and found that the RRF area in pain patients were enlarged compared with pain-free volunteers. ${ }^{6,38}$ In a recent study, we unexpectedly found larger RRF areas in patients with acute low back pain compared with chronic low back pain. ${ }^{6}$ However, due to small group sizes, we were unable to perform an analysis that accounted for confounding factors. Therefore, we performed a study of 214 patients to investigate the discriminative ability of RRF to distinguish patients with acute and chronic low back pain. The aim was to explore the potential role of expansion of RRF area in the transition to chronic pain. We expected that the development of chronic pain would be associated with enlargement of RRF areas due to central sensitization processes. Unlike previous studies, we performed multivariable analyses to control for sociodemographic and clinical characteristics that might be associated with the transition from acute to chronic low back pain.

\section{Methods}

\subsection{Participants}

We screened and examined patients with acute and chronic low back pain for eligibility according to a common protocol at the Department of Anesthesiology and Pain Medicine of the University of Bern, a single tertiary care university center specialized in pain medicine. Patients with acute low back pain were referred by a local primary care group practice, patients with chronic low back pain by the departments of orthopedics and neurosurgery of the University Hospital of Bern and the Orthopedic Center Sonnenhof in Bern. Acute low back pain was defined as average lumbar back pain of at least 3 on a numeric rating scale (NRS) where 0 indicated "no pain" and 10 "worst pain imaginable" at any day during the week preceding recruitment, with a maximal duration of 6 weeks and no more than 3 pain episodes during the last year. Chronic low back pain was defined as lumbar back pain of at least 3 on the NRS at most days during the week preceding recruitment, with a minimal duration of 3 months. Patients were excluded if they suffered from an acute lumbosacral radiculopathy, rheumatologic inflammatory disease, neurological comorbidity potentially affecting the neurological function of the lower extremity to be tested, or psychiatric comorbidity except unipolar depressive disorder. We also excluded patients unable to understand the consequences of study participation due to language problems, patients who could not be reached by phone and mail, and pregnant women. A history of back surgery or of accident leading to the back pain and pain not satisfying our definition of acute low back pain were additional exclusion criteria for patients with acute low back pain. A history of instrumented surgery, multilevel degenerative changes with planned surgery of more than 3 segments, bilateral pain below both knees, isolated leg pain and pain not satisfying our definition of chronic low back pain were additional exclusion criteria for patients with chronic low back pain. We performed all tests according to a prospective protocol approved by the local research ethics committee and in accordance with the Declaration of the World Medical Association. ${ }^{56}$ All patients gave written informed consent. The patients analyzed in this study were completely unrelated to our previous publications. ${ }^{6,37}$

\subsection{Clinical and radiologic assessment}

Before inclusion into the study, patients with acute low back pain underwent physical examination by their referring general practitioner. In case of suspected acute lumbosacral radiculopathy due to a herniated disc, patients would also receive a magnetic resonance image (MRI) in accordance with clinical guidelines, ${ }^{9}$ but none of the patients was reported to have received an $\mathrm{MRI}$. Patients with chronic low back pain underwent a detailed orthopedic and neurological assessment by the referring orthopedic surgeon or neurosurgeon and imaging of the lumbar spine by either computer tomography or MRI before inclusion into the study. All images were centrally read by an independent musculoskeletal radiologist at University Hospital Zürich, for details see web-appendix a (available online as Supplemental Digital Content at http://links.Iww.com/PAIN/ A325). At study inclusion, all patients underwent a repeat physical examination by study staff to identify any sensory or motor deficit of the lower extremity.

\subsection{Assessments of RRF}

We performed the RRF assessments according to our previously described methodology ${ }^{36}$ and selected the foot contralateral to the side of most pain for the assessment. In case of bilateral back pain, the side was randomly selected according to a computergenerated list. We placed 10 cathodal surface electrodes $(15 \times$ $15 \mathrm{~mm}$, type 700 Ambu AVS, Denmark) at the sole of the foot and a common anode (50 $\times 90 \mathrm{~mm}$, type Pals, United States) on the dorsum of the foot. Each stimulus consisted of a train of 5 individual constant-current pulses, each pulse lasting 1 millisecond, delivered at $200 \mathrm{~Hz}$ (Stimulator Noxitest IES 230, Aalborg, Denmark); the stimulus was perceived as single stimulus by the participants. A training session enabled all patients to get familiar with the electrical stimulation.

In a first step, we determined pain detection thresholds, defined as current intensity at which pain sensation can be evoked, using a staircase assessment method at each of the 10 electrodes. To avoid the subjects' adaptation to the stimulus while determining the pain threshold at each stimulation site, we repeatedly presented the identified pain threshold at the reference electrode located in the middle of the medial longitudinal arch of the foot to the participant. This guaranteed equally intensive stimulations and thus equal probability for an NWR to be elicited at all stimulation sites. After determination of pain detection thresholds, we multiplied the corresponding stimulus intensity at each by a factor 1.5 to ensure stimulation intensity sufficient to reliably evoke a reflex response of the tibialis anterior muscle. Finally, the recording phase begun, during which we registered evoked pain intensity and NWR amplitudes. We stimulated every electrode 5 times in a random sequence using a computer-controlled electrical relay resulting in a total of 50 stimuli. Study participants rated pain intensity for each stimulation using a 10-cm electronic visual analogue scale (Aalborg University, Denmark), where 0 indicated "no pain" and 10 "worst pain imaginable." We recorded the electromyogram with surface electrodes (Type 720; Ambu AVS, Denmark) over the belly of the tibialis anterior muscle. The electromyographic signals were amplified (up to 50'000 times), filtered (5-500 Hz, second order), sampled $(2000 \mathrm{~Hz})$, and stored from 200 milliseconds before until 1000 milliseconds after stimulation to ensure that the reflex sensitive time interval of 60 to 180 milliseconds after stimulation was recorded. We finally calculated the area of the RRF according to our previously established method. ${ }^{36}$ In summary, we quantified the NWR sizes at all stimulation sites using rootmean-square (RMS) amplitudes in the 60- to 180-millisecond poststimulation interval. We then derived spatial maps of NWR on the sole of the foot by 2-dimensional interpolation of the RMS amplitudes. As in our previous publications, ${ }^{35,36}$ we calculated the RRF area as the fraction of the foot sole delimited by 
a threshold set by the peak RMS amplitude (largest reflex irrespective of stimulation site) minus 2 times the standard deviation of the remaining RMS amplitudes. This method has shown good reproducibility. ${ }^{27}$

\subsection{Assessment of sociodemographic, clinical, and psychological characteristics}

We assessed sociodemographic, clinical, and psychological characteristics in all patients and recorded the following covariates: age ( $\leq 40,41-60$, and $\geq 60$ years); gender; education (higher education vs lower education); living status (living alone vs not living alone); body mass index (overweight vs normal weight); pain intensity (high vs low baseline pain); depression (yes vs no) and anxiety disorder (yes vs no); regular intake of nonsteroidal anti-inflammatory drugs, paracetamol, metamizole, opioides, antidepressants, and anticonvulsants (yes vs no). We considered patients with college or university degree as having higher education and defined overweight as a body mass index (BMI) $\geq 25 \mathrm{~kg} / \mathrm{m}^{2}$ and high baseline pain as pain of at least NRS 5 . We used the Beck Depression Inventory version $2^{34}$ and the State-Trait Anxiety Inventory ${ }^{30}$ to assess depressive or anxiety disorders; we defined depression as a Beck Depression Inventory $\geq 13$, and anxiety as a State-Trait Anxiety Inventory Trait $t$-value $\geq 60$. We chose these parameters because of their documented prognostic value, based on previous studies and systematic reviews. ${ }^{8,10,13,14,20-24,28,31,40,47,48,51}$ We had prespecified to dichotomize BMI, pain intensity, depression, and anxiety to facilitate a clinically meaningful interpretation and to ensure comparability of regression coefficients for all covariates.

\subsection{Statistical analysis}

We based our sample size calculation on the pooled standard deviation of 0.197 of the RRF area of patients with acute and chronic low back pain. ${ }^{6}$ According to this sample size calculation, a comparison of 110 patients with acute low back pain and 110 patients with chronic low back pain detects a mean difference in the RRF area between the 2 groups of at least 0.07 with a power of 0.8 at a 2 -sided alpha of 0.05 .

We assessed the discriminative ability of the RRF to distinguish patients with acute and chronic low back pain by comparing RRF areas between groups as continuous fraction of the foot sole using linear regression, and after dichotomization using logistic regression. We reported differences between groups in mean values and odds ratios, respectively. The RRF area was dichotomized into large and small areas according to normative data collected in a population of 300 healthy individuals, ${ }^{35}$ using the 75 th percentile at 0.62 as cutoff. We used univariable models, then performed age- and sex-adjusted analyses and finally fitted fully adjusted multivariable models to account for the abovementioned sociodemographic and clinical confounders for which it was plausible that they could be related to a transition from acute to chronic low back pain. ${ }^{37}$ The univariable and multivariable association of these variables with type of pain (acute vs chronic) were determined using logistic regression.

We performed 2 prespecified sets of sensitivity analyses of the difference in RRF areas between acute and chronic low back pain patients. First, we used multivariable models that omitted depression and anxiety as covariates since the cross-sectional design of the study would not allow to rule out reverse causality. Second, we restricted analyses to patients reporting a pain intensity of at least NRS 3 at the reference electrode and in whom reported pain intensity was similar at all 10 electrodes after electrical stimulation. Patients who suffer from a painful condition at the time of the experiment appeared less compliant with the experimental protocol than healthy controls despite careful instructions. We therefore found it difficult in some patients to achieve sufficient stimulation intensity, which was similar across all 10 electrodes of the electrical stimulation used to evoke an NWR. In a sensitivity analysis, we therefore included patients only if they reported that the perceived pain intensity after stimulation was at least NRS 3 at the reference electrode and if the perceived pain across all electrodes was similar as defined by an intraclass correlation coefficient larger than 0.60. The intraclass correlation was defined as the variance of the pain intensity at the reference electrode across patients divided by the sum of the variances across patients and across electrodes within patients. In additional 3 sets of sensitivity analyses performed post hoc we (1) adjusted for types of pharmacological treatment; (2) incorporated age, pain intensity, depression, and anxiety as continuous covariates; and (3) excluded patients with acute low back pain at baseline with persistent low back pain 6 months after recruitment. All analyses were performed with Stata (Version 12.1; StataCorp, College Station, TX) or MATLAB (Version 8.3; The MathWorks Inc, Natick, MA).

\section{Results}

We totally screened 960 patients with chronic low back pain from 2012 to 2015 and screened 551 patients with acute low back pain from 2009 and 2015 for study participation. We successfully included 113 chronic and 101 acute low back pain patients (Fig. 1). The most important reason for exclusion was denial of study participation: $21 \%$ of all eligible chronic and $29 \%$ of all eligible acute low back pain patients were not willing to participate in the study. Other important reasons for exclusion in patients with chronic low back pain were a history of instrumented back surgery (20\%) or planned multilevel surgery on more than 3 segments (18\%). Low pain intensity at baseline, defined as pain less than 3 on the NRS, was an important reason for exclusion of patients with acute low back pain (18\%) but not of patients with chronic low back pain (2\%). The second most important reason for exclusion in patients with acute low back pain was persistence of pain for longer than 6 weeks and thus not classifying as acute pain according to our definition (18\%). Approximately $6 \%$ of all patients with chronic and $3 \%$ of all patients with acute low back pain could not be reached by phone and mail. We could not perform the RRF assessment in 4\% of the patients with chronic and $7 \%$ of patients with acute low back pain due to defect of the RRF equipment. The patients not being reached and not being tested due to RRF equipment out of order did not differ in sociodemographic and clinical characteristics from the patients included in the study (data not presented). Patients with acute low back pain did not receive imaging in their diagnostic work up. ${ }^{9}$ A total of 109 (96\%) patients with chronic low back pain received imaging, 104 patients had an MRl and 5 patients had a computed tomography. Web-appendix a presents measures of the severity of degenerative changes in these patients (available online as Supplemental Digital Content at http://links.lww.com/PAIN/A325).

Table 1 shows the baseline characteristics of patients according to their pain status. Mean age in patients with acute low back pain was 43.6 years (SD, 13.9 years) and in patients with chronic low back pain 61.7 years (SD, 14.3 years). From all patients with acute and chronic low back pain, 47 (47\%) and 69 (61\%) were women, respectively. A higher proportion of patients with acute low back pain had higher education and was living alone. Mean pain at baseline was 6.3 (SD, 2.0) in patients with acute and 6.3 (SD, 1.4) in 


\begin{tabular}{|c|c|c|c|c|c|}
\hline \multicolumn{5}{|l|}{ CLBP patients eligible $\quad \mathrm{N}=960$} & ALBP patients eligible: $\mathrm{N}=\mathbf{5 5 1}$ \\
\hline & CLBP patients excluded & $\mathrm{N}=\mathbf{8 4 8}$ & \multicolumn{2}{|l|}{ ALBP patients excluded } & \\
\hline & No chronic pain & $\mathrm{N}=8$ & No acute pain & $\mathrm{N}=82$ & \\
\hline & Pain at recruitment $<3 \mathrm{NRS}^{\circ}$ & $\mathrm{N}=18$ & Pain at recruitment $<3 \mathrm{NRS}^{\circ}$ & $\mathrm{N}=57$ & \\
\hline & Rheumatologic disease & $\mathrm{N}=48$ & Rheumatologic disease & $\mathrm{N}=10$ & \\
\hline & Neurologic or psychiatric co-morbidity & $\mathrm{N}=96$ & Neurologic or psychiatric co-morbidity & $\mathrm{N}=65$ & \\
\hline & - Polyneuropathy & $\mathrm{N}=52$ & - Polyneuropathy & $\mathrm{N}=5$ & \\
\hline & - Herniated disc & $\mathrm{N}=2$ & - Herniated disc & $\mathrm{N}=57$ & \\
\hline & $\begin{array}{ll}\text { - } & \text { Parkinson disease } \\
\text { - Bilateral sensory/motor loss legs }\end{array}$ & $\begin{array}{l}\mathrm{N}=11 \\
\mathrm{~N}=10\end{array}$ & - Psychiatric co-morbidity & $\mathrm{N}=3$ & \\
\hline & - Residual symptoms after stroke & $\mathrm{N}=9$ & & & \\
\hline & $\begin{array}{l}\text { - Other neurological co-morbidity } \\
\text { - Psychiatric co-morbidity }\end{array}$ & $\begin{array}{l}\mathrm{N}=6 \\
\mathrm{~N}=6\end{array}$ & & & \\
\hline & Instrumented previous back surgery & $\mathrm{N}=173$ & Any previous back surgery & $\mathrm{N}=10$ & \\
\hline & $>3$ segmental surgery planned & $\mathrm{N}=149$ & History of accident & $\mathrm{N}=21$ & \\
\hline & Bilateral pain below both knees & $\mathrm{N}=60$ & & & \\
\hline & Isolated leg pain & $\mathrm{N}=8$ & & & \\
\hline & Could not be reached & $\mathrm{N}=47$ & Could not be reached & $\mathrm{N}=15$ & \\
\hline & Participation denied & $\mathrm{N}=179$ & Participation denied & $\mathrm{N}=132$ & \\
\hline & RRF equipment out of order & $\mathrm{N}=31$ & RRF equipment out of order & $\mathrm{N}=30$ & \\
\hline$\downarrow$ & Other & $\mathrm{N}=31$ & Other & $\mathrm{N}=27$ & 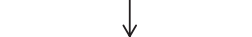 \\
\hline CLBP patients included $\mathrm{N}=112$ & & & & & ALBP patients included $N=102$ \\
\hline
\end{tabular}

Figure 1. Flow chart of inclusion and exclusion of patients with chronic low back pain (CLBP) and acute low back pain (ALBP). Other CLBP: 6 polymorbidity, 25 unclear. Other ALBP: 2 primary care physicians denied contact with patient, 9 pregnancy, 8 language problems, 8 unclear. NRS, numeric rating scale; RRF, reflex receptive field.

patients with chronic low back pain. The mean BMI was $25.1 \mathrm{~kg} / \mathrm{m}^{2}$ (SD, 4.3) and 28.1 (SD, 4.4), respectively.

In crude, univariable analyses, we found significantly increased risk of chronic low back pain in patients with higher age, female gender, high baseline pain, overweight, and comorbid depressive disorder. In adjusted multivariable analyses, the risk of suffering from chronic low back pain was unaffected by gender $(P=0.16)$, high baseline pain ( $P=0.68)$, overweight $(P=0.10)$, and anxiety $(P=0.28)$. Age was independently associated with an increased risk of chronic low back pain $(P<0.001)$, as was concomitant depressive disorder $(P=0.001)$. Conversely, high education and living alone were independently associated with a decreased risk of chronic low back pain in multivariable logistic regression analysis $(P=0.01$ and $P=0.002$, respectively). Web-appendix b (available online as Supplemental Digital Content at http://links. Iww.com/PAIN/A325) shows a comparison of pharmacological treatments between patients with acute and chronic low back pain. Patients with acute low back pain were more likely to take nonsteroidal anti-inflammatory drugs compared with patients with chronic low back pain, but less likely to take antidepressants or anticonvulsants.

Figure 2 shows average RRF in patients with acute (left) and chronic (right) low back pain: the RRF areas were similar, with mean values of 0.34 (SD, 0.19) and 0.33 (SD, 0.21), respectively. Table 2 shows the corresponding crude and adjusted estimates of the differences in mean RRF areas (left) and odds ratios of enlarged RRF (right) in patients with acute and chronic low back pain. There was no evidence for a difference in RRF areas

\section{Table 1}

\section{Characteristics of patients with chronic low back pain and acute low back pain at baseline $(\mathrm{N}=214)$.}

\begin{tabular}{|c|c|c|c|c|c|c|}
\hline & CLBP $(\mathrm{N}=112)$ & ALBP $(\mathrm{N}=102)$ & Univariable OR $(95 \% \mathrm{CI})$ & $P$ & Multivariable OR (95\% Cl) & $P$ \\
\hline \multicolumn{7}{|l|}{ Sociodemographic characteristics } \\
\hline \multicolumn{7}{|l|}{ Age, y } \\
\hline$\leq 40$ & $9(8)$ & $46(45)$ & 1 (Reference) & & 1 (Reference) & \\
\hline $41-60$ & $41(37)$ & $42(41)$ & $5.00(2.17-11.49)$ & & $3.21(1.26-8.18)$ & \\
\hline$>60$ & $62(55)$ & $14(14)$ & $22.63(9.02-56.81)$ & $<0.001^{*}$ & $18.11(6.33-51.77)$ & $<0.001^{*}$ \\
\hline Females & $68(61)$ & $47(46)$ & $1.81(1.05-3.11)$ & 0.03 & $1.72(0.81-3.63)$ & 0.16 \\
\hline Higher education & $26(23)$ & $47(46)$ & $0.35(0.20-0.64)$ & 0.001 & $0.38(0.18-0.82)$ & 0.01 \\
\hline Living alone & $19(17)$ & $30(30)$ & $0.49(0.26-0.94)$ & 0.03 & $0.22(0.08-0.56)$ & 0.002 \\
\hline \multicolumn{7}{|l|}{ Clinical characteristics } \\
\hline $\begin{array}{l}\text { High average pain at baseline (NRS cutoff } \\
\geq 5 \text { ) }\end{array}$ & $97(87)$ & $77(76)$ & $2.10(1.04-4.26)$ & 0.04 & $1.23(0.46-3.33)$ & 0.68 \\
\hline Overweight (BMl cutoff $\geq 25 \mathrm{~kg} / \mathrm{m}^{2}$ ) & $78(70)$ & $41(40)$ & $3.41(1.94-6.00)$ & $<0.001$ & $1.91(0.89-4.11)$ & 0.10 \\
\hline Depression (BDI-II cutoff $\geq 13$ ) & $36(32)$ & $13(13)$ & $3.24(1.60-6.56)$ & 0.001 & $6.92(2.17-22.05)$ & 0.001 \\
\hline Anxiety (STAl Trait cutoff $\geq 60$ ) & $24(21)$ & $15(15)$ & $1.58(0.78-3.22)$ & 0.20 & $0.54(0.17-1.66)$ & 0.28 \\
\hline
\end{tabular}

OR >1.0 means increased risk for chronic low back pain. Values are $\mathrm{n}(\%)$, crude and adjusted OR with corresponding 95\% Cls and $P$ values from univariable and multivariable logistic regression models. * $P$-value for trend.

BDI-II, Beck Depression Inventory Version 2; Cl, confidence interval; BMI, body mass index; NRS, Numerical Rating Scale from 0 (no pain) to 10 (maximum pain); OR, odds ratio; STAI Trait, State Trait Anxiety Index. 


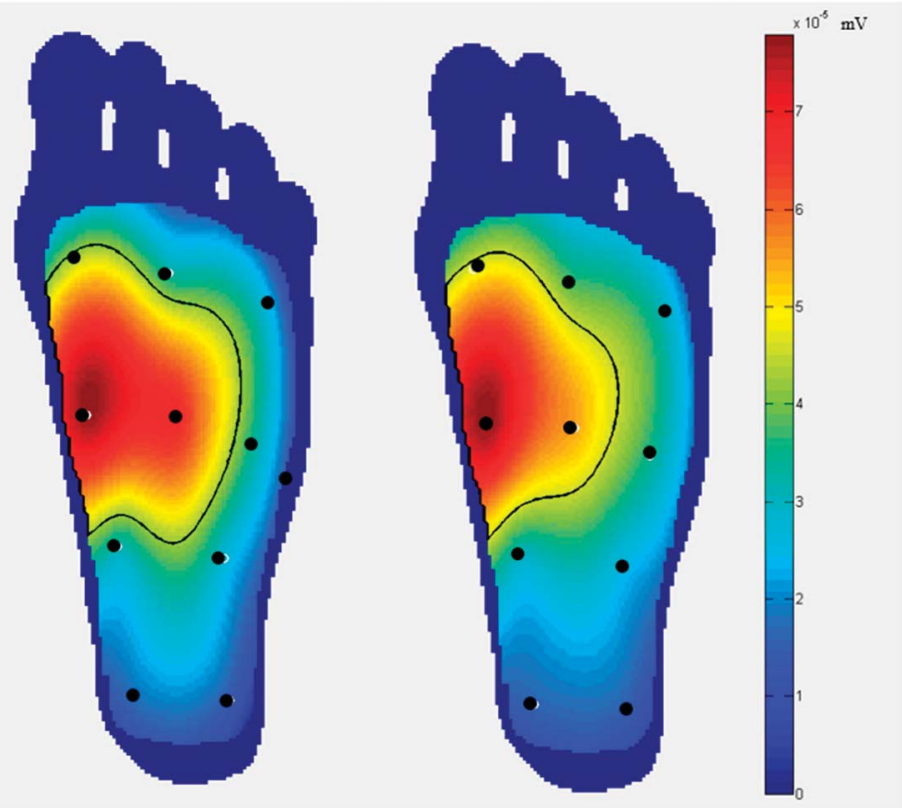

Figure 2. Reflex Receptive Field (RRF) areas in patients with chronic and acute low back pain ( $\mathrm{N}$ total $=214$ ). Mean RRF obtained by averaging the nociceptive withdrawal reflex responses from patients with acute low back pain (left) and chronic low back pain (right) depicting the RRF of an average subject for each group. Black dots indicate stimulation sites; black lines define the contour of the RRF area as fraction of the sole of the foot; colors depict reflex magnitude evoked at the sole of the foot.

between groups neither in crude nor in any of the adjusted analyses; 95\% confidence intervals (Cls) for mean differences (left) included zero as measure of no effect and for odds ratios (right) included 1 as measure of no effect that corresponds to nonsignificant $P$-values for all analyses. The 95\% Cls ruled out a clinically relevant difference in mean RRF areas between acute and chronic low back pain patients.

This lack of evidence in the main analysis for a difference in RRF areas between acute and chronic low back pain patients was confirmed in all sensitivity analyses. Not adjusting for depression or anxiety or for both variables in the full model resulted in mean differences of $-0.03(95 \% \mathrm{Cl},-0.09$ to 0.04$),-0.02(95 \% \mathrm{Cl}$, -0.88 to 0.05$)$, or -0.03 ( -0.10 to 0.03 ), and odds ratios of 1.04 (95\% Cl, 0.37-2.87), 1.10 (95\% Cl, 0.39-3.07), or 0.92 (95\% Cl, $0.34-2.47)$, respectively. Table 3 shows results from univariable and multivariable sensitivity analyses restricted to those 113 patients with sufficient and similar nociceptive input during the experimental procedure after exclusion of 56 patients with acute and 45 patients with chronic low back pain; results were again similar to the main analyses, but with wider Cls for the odds ratios.
Web-appendix $b$ presents the association of pharmacological treatments with dichotomous and continuous estimates of RRF areas, with significant associations found between metamizole and opioids and dichotomized areas of RRF and between metamizole and continuous areas of RRF (available online as Supplemental Digital Content at http://links.Iww.com/PAIN/ A325). However, we again found no differences in estimates of RRF areas between patients with acute and chronic low back pain after including pharmacological treatments as covariates. Web-appendix c presents the difference in estimates of RRF areas after adjustment for age, pain intensity, depression, and anxiety severity as continuous covariates, again with no differences in RRF between groups of patients (available online as Supplemental Digital Content at http://links.Iww.com/PAIN/ A325). Web-appendix $d$ shows results from univariable and multivariable sensitivity analyses after exclusion of 13 patients with acute low back pain who presented with persistent low back pain 6 months after recruitment; again, we found no differences in RRF areas (available online as Supplemental Digital Content at http://links.lww.com/PAIN/A325).

\section{Table 2}

Main analysis of differences of RRF between patients with acute and chronic low back pain ( $N=214)$.

\begin{tabular}{|c|c|c|c|c|}
\hline & \multicolumn{2}{|l|}{ RRF area continuous* } & \multicolumn{2}{|c|}{ RRF area dichotomised† } \\
\hline & Mean difference $(95 \% \mathrm{CI})$ & $P$ & Odds ratio $(95 \% \mathrm{CI})$ & $P$ \\
\hline Crude model & $-0.02(-0.07$ to 0.04$)$ & 0.55 & $0.90(0.40-2.04)$ & 0.80 \\
\hline Age- and sex-adjusted model & $-0.03(-0.09$ to 0.04$)$ & 0.42 & $0.98(0.38-2.54)$ & 0.98 \\
\hline Fully adjusted modelł & $-0.02(-0.09$ to 0.04$)$ & 0.51 & $1.08(0.38-3.06)$ & 0.88 \\
\hline
\end{tabular}

* inear regression on the normal scale with RRF area as fraction of the foot sole considered as continuous outcome and group difference as mean difference (MD) between RRF areas. MD <1.0 means smaller RRF areas in patients with chronic low back pain.

† Logistic regression with RRF area as binary outcome (cutoff: mean RRF area $\leq 0.62$ ) and group difference as OR comparing large vs small RRF area. OR $<1.0$ means lower risk of expanded RRF areas in patients with chronic low back pain.

$\ddagger$ Adjusted for age, sex, education, living status, average pain at baseline, body mass index, depression, and anxiety.

$\mathrm{Cl}$, confidence interval; OR, odds ratio; RRF, reflex receptive field. 
Table 3

Sensitivity analysis of differences of RRF between patients with acute and chronic low back pain restricted to subjects with intensive and similar nociceptive input during the experiment $(\mathrm{N}=113)$.

\begin{tabular}{|c|c|c|c|c|}
\hline & \multicolumn{2}{|l|}{ RRF area continuous* } & \multicolumn{2}{|c|}{ RRF area dichotomised } \\
\hline & Mean difference (95\% Cl) & $P$ & $\overline{\mathrm{OR}}(95 \% \mathrm{CI})$ & $P$ \\
\hline Crude model & $-0.04(-0.11$ to 0.04$)$ & 0.31 & $0.92(0.27-3.10)$ & 0.89 \\
\hline Age and sex-adjusted model & $-0.03(-0.12$ to 0.06$)$ & 0.54 & $1.33(0.31-5.79)$ & 0.70 \\
\hline Fully adjusted model & $-0.03(-0.13$ to 0.07$)$ & 0.54 & $1.81(0.32-10.32)$ & 0.88 \\
\hline
\end{tabular}

* Linear regression on the normal scale with RRF area as fraction of the foot sole considered as continuous outcome and group difference as mean difference (MD) between RRF areas. MD $<1.0$ means smaller RRF areas in patients with chronic low back pain.

† Logistic regression with RRF area as binary outcome (cutoff: mean RRF area $\leq 0.62$ ) and group difference as OR comparing large vs small RRF area. OR <1.0 means lower risk of expanded RRF areas in patients with chronic low back pain.

$\ddagger$ Adjusted for age, sex, education, living status, average pain at baseline, body mass index, depression, and anxiety.

$\mathrm{Cl}$, confidence interval; OR, odds ratio; $\mathrm{RRF}$, reflex receptive fields.

\section{Discussion}

\subsection{Main findings}

The discriminative ability of the RRF to distinguish between patients with acute and chronic low back pain was poor in this large-scale analysis. We found no difference in RRF areas between groups, neither in crude nor adjusted analyses. If we considered the RRF as continuous explanatory variable and thus the point estimates correspond to the mean differences between patients with acute and chronic low back pain, crude and adjusted point estimates numerically suggest a slightly smaller mean RRF area in patients with chronic low back as previously found. ${ }^{6}$ However, the difference was minute and $95 \% \mathrm{Cl}$ excluded any clinically relevant difference between groups in either direction. Similarly, if we considered the RRF as binary variable, the estimated odds ratios suggested that patients with chronic low back pain were less likely to display enlarged RRF areas as compared with patients with acute low back pain. For these analyses, 95\% Cl were wide and could not rule out clinically relevant differences between groups with possibly increased or decreased odds ratios for enlarged RRF areas in patients with chronic low back pain. The lack of evidence for a difference in RRF area between patients with acute and chronic low back pain was also observed in both sensitivity analyses. Conversely, we found pronounced differences in age, education, living status, and depression between groups. Older patients and patients suffering from concomitant depression had an increased risk for chronic low back pain, whereas patients with higher education or living alone were less likely to suffer from chronic pain.

\subsection{Strengths and limitations}

This is the first large-scale application of RRF as novel method to objectively assess altered central pain processing in patients with low back pain. Our group developed the method, applied it first in healthy volunteers, ${ }^{36}$ and then tested it in case-control studies comparing chronic pain patients with pain-free individuals. ${ }^{7,39}$ The current study included totally 214 patients and investigated the discriminative ability of RRF to distinguish patients with acute and chronic low back pain and thereby represents an important translational research step. RRF is a method to objectively explore mechanisms of altered central pain processing, because it is based on the detection of NWR, an involuntary reaction to painful stimulation. Therefore, the method may be particularly useful to assess altered central pain processing compared with other quantitative sensory tests that mostly rely on the subjective response of the study participant.

Acute low back pain patients were referred to us by a primary care group practice as the usual point of care for this type of patients. Chronic low back pain patients were referred by surgical departments of 2 hospitals of the same city, again reflecting the main point of care for patients with longlasting low back pain. This 2-gate design is widely applied in diagnostic case-control studies, because it reflects the reality of distinct referral patterns for nondiseased and diseased patients, ${ }^{29,44}$ or, as in our case, patients with acute and chronic low back pain. All patients were screened for eligibility and examined according to a common prospective protocol by a single tertiary care university center specialized in pain medicine. It is in the nature of the design that differences between groups tend to be inflated and that some of the observed differences between groups may be partially explained by differences in referral pathways than the pain status of acute vs chronic low back pain. However, this was the case only for some sociodemographic, clinical, and psychological baseline characteristics. The large sample size allowed us to adjust for a number of baseline characteristics possibly involved in the transition from acute to chronic low back pain and thus acting as potential confounders. Despite the tendency of inflating differences between groups in the present study, we were unable to find any evidence for a difference in RRF areas. The large sample size and associated statistical precision and the 2-gate design of our study make it likely that we would have detected a difference if it had been present.

Because the data collection was cross-sectional rather than longitudinal, the direction of the causal pathway will remain unclear for some variables. We were therefore unable to rule out reverse causality for depression or anxiety and performed adjusted sensitivity analyses after dropping these variables as covariates; the results did not change. Data quality was excellent throughout, with only a small amount of missing data for the covariates. An unexpected limitation of our study was the difficulty encountered in the experimental set-up in pain patients who appeared less compliant with the experimental protocol than healthy controls, despite careful instructions. Because this could have biased results toward the null, we performed an additional sensitivity analysis restricted to patients who reported a pain intensity of at least 3 on a NRS and in whom the variation in perceived pain intensity across all 10 electrodes was low, and found our main results confirmed.

\subsection{Context}

The assessment of the spinal withdrawal reflex in response to nociceptive stimulation is accepted as one of the most robust measure of central sensitization processes, and thus RRF measurement is a promising method to objectively assess spinal nociceptive hyperexcitability in humans. ${ }^{2,26,33,45}$ We performed 
2 case-control studies comparing pain patients with pain-free individuals and found an expansion of RRF in patients, indicating that expansion of RRF areas occurs in patients with both acute and chronic pain conditions including low back pain. ${ }^{6,38}$ At the spinal cord level, the RRF is most likely organized by widedynamic range neurons located in the deep dorsal horn. ${ }^{12,46}$ Thus, the expansion of RRF areas is likely to express spinal nociceptive hyperexcitability and could be a consequence of increased number of responsive spinal neurons or increased synaptic sensitivity. ${ }^{1}$

However, to decide on the usefulness of the method for future use in clinical practice, the relevant question is not if the RRF can be used to distinguish pain patients from pain-free persons, but to determine its discriminative ability to distinguish patients with acute and chronic pain. We therefore performed the current study and found no difference in RRF areas between patients with acute and chronic low back pain.

When we designed the study, we expected that RRF expansion would occur with transition to chronic pain, possibly as a result of central sensitization processes. As expansion leads to amplification of nociceptive signals within the central nervous system, this phenomenon would be one of the mechanisms explaining persistent pain. Our expectation was not confirmed. A possible explanation for this finding is that expansion of RRF areas develops early in the course of a pain syndrome, rather than gradually at the transition to a chronic condition. As mentioned above, we previously found enlarged RRF in acute low back pain compared with pain-free subjects. ${ }^{6}$ The results of the present study suggest that RRF expansion does not progress as the condition becomes chronic. Rather than being involved in the transition to chronic pain, expansion of RRF may be present and contribute to pain throughout the course of low back pain. Support to this explanation can be found in a previous longitudinal study in whiplash patients, which showed that spinal nociceptive hyperexcitability (as detected by lowered NWR thresholds) were present early after injury, persisted in those patients who developed chronic pain, but resolved in those patients who recovered. ${ }^{50}$ The RRF may follow a similar time course, which would explain our results.

\subsection{Implications}

Our study indicates that it is necessary to simplify the experimental protocol and to take measures to standardize the nociceptive input. We took first steps toward this and tested the adapted protocol in 21 healthy volunteers. ${ }^{27}$ This recent study suggested quantifying the RRF based on the NWR thresholds detected at each stimulation site. The novel methodology showed good reliability but is yet to be applied to pain patients.

Although expansion of RRF area is unlikely to be involved in the transition from acute to chronic low back pain, we are unable to conclude that RRF areas have no predictive value to identify patients at risk of developing chronic pain. A longitudinal study set-up using a cohort of patients with acute low back pain followed up for several months to detect incident cases of chronic low back pain would be necessary to investigate predictors of the transition from acute to chronic low back pain.

\section{Conclusion}

The discriminative ability of the RRF to distinguish between patients with acute and chronic low back pain is poor. This suggests that expansion of RRF area is unlikely to be involved in the transition from acute to chronic low back pain. We found different sociodemographic and psychological characteristics to be associated with chronic low back pain. Further studies should aim at simplifying and standardizing the RRF assessment method and should determine the prognostic value of RRF to identify patients at risk of developing chronic pain.

\section{Conflict of interest statement}

The authors have no conflicts of interest to declare.

The study was funded by the Swiss National Science Foundation (No. 3247BO_122358/1 and No. 32003B_138361) and the Scientific Funds of the University Department of Anesthesiology and Pain Therapy of the University of Bern.

M. Müller and M. Curatolo conceived the study. M. Müller, F. Treichel, and P. Heini contributed to data collection. J.A. Biurrun Manresa and O.K. Andersen developed the methodology for the RRF assessment. M. Müller and P. Jüni did the data preparation and analysis. All authors participated in interpretation of the data. M. Müller, M. Curatolo, and P. Jüni wrote the first draft of the paper and all authors contributed to the final draft.

Previous presentation of the research: The results of this study were not presented at any conference nor was the manuscript under consideration by any other journal.

\section{Appendix A. Supplemental Digital Content}

Supplemental Digital Content associated with this article can be found online at http://links.Iww.com/PAIN/A325.

\section{Article history:}

Received 13 April 2016

Received in revised form 10 July 2016

Accepted 25 July 2016

Available online 29 July 2016

\section{References}

[1] Andersen OK, Biurrun Manresa JA. Electrophysiological techniques: assessment of spinal nociceptive processing using the withdrawal reflex. In: Postoperative Pain, Wilder-Smith OH, Arendt-Nielsen L, Yarnitsky D, Visser KCP, editors. Philadelphia: Wolters Kluwer Deutschland, 2015. p 109-18.

[2] Andersen OK, Sonnenborg FA, Arendt-Nielsen L. Modular organization of human leg withdrawal reflexes elicited by electrical stimulation of the foot sole. Muscle Nerve 1999;22:1520-30.

[3] Andersen OK, Sonnenborg FA, Arendt-Nielsen L. Reflex receptive fields for human withdrawal reflexes elicited by non-painful and painful electrical stimulation of the foot sole. Clin Neurophysiol 2001;112:641-9.

[4] Andersson GB. Epidemiological features of chronic low-back pain. Lancet 1999;354:581-5.

[5] Banic B, Petersen-Felix S, Andersen OK, Radanov BP, Villiger PM, ArendtNielsen L, Curatolo M. Evidence for spinal cord hypersensitivity in chronic pain after whiplash injury and in fibromyalgia. PAIN 2004;107:7-15.

[6] Biurrun Manresa JA, Neziri AY, Curatolo M, Arendt-Nielsen L, Andersen OK. Reflex receptive fields are enlarged in patients with musculoskeletal low back and neck pain. PAIN 2013;154:1318-24.

[7] Bjerre L, Andersen AT, Hagelskjaer MT, Ge N, Morch CD, Andersen OK. Dynamic tuning of human withdrawal reflex receptive fields during cognitive attention and distraction tasks. Eur J Pain 2011;15:816-21.

[8] Carey TS, Garrett JM, Jackman AM. Beyond the good prognosis. Examination of an inception cohort of patients with chronic low back pain. Spine (Phila Pa 1976) 2000;25:115-20.

[9] Chou R, Qaseem A, Snow V, Casey D, Cross JT Jr, Shekelle P, Owens DK. Diagnosis and treatment of low back pain: a joint clinical practice guideline from the American College of Physicians and the American Pain Society. Ann Intern Med 2007;147:478-91.

[10] Chou R, Shekelle P. Will this patient develop persistent disabling low back pain? JAMA 2010;303:1295-302.

[11] Clarke RW, Ford TW, Taylor JS. Reflex actions of selective stimulation of sural nerve C fibres in the rabbit. Q J Exp Physiol 1989;74:681-90. 
[12] Cook AJ, Woolf CJ, Wall PD, McMahon SB. Dynamic receptive field plasticity in rat spinal cord dorsal horn following C-primary afferent input. Nature 1987;325:151-3.

[13] Costa Lda C, Maher CG, McAuley JH, Hancock MJ, Herbert RD, Refshauge KM, Henschke N. Prognosis for patients with chronic low back pain: inception cohort study. Br Med J 2009;339:b3829.

[14] Coste J, Lefrancois G, Guillemin F, Pouchot J. Prognosis and quality of life in patients with acute low back pain: insights from a comprehensive inception cohort study. Arthritis Rheum 2004;51:168-76.

[15] Curatolo M. Diagnosis of altered central pain processing. Spine (Phila Pa 1976) 2011;36(25 suppl I):S200-204.

[16] Dagenais S, Caro J, Haldeman S. A systematic review of low back pain cost of illness studies in the United States and internationally. Spine J 2008;8:8-20.

[17] Ekman M, Jonhagen S, Hunsche E, Jonsson L. Burden of illness of chronic low back pain in Sweden: a cross-sectional, retrospective study in primary care setting. Spine (Phila Pa 1976) 2005;30:1777-85.

[18] France CR, France JL, al'Absi M, Ring C, Mclntyre D. Catastrophizing is related to pain ratings, but not nociceptive flexion reflex threshold. PAIN 2002;99:459-63.

[19] France CR, Keefe FJ, Emery CF, Affleck G, France JL, Waters S, Caldwell DS, Stainbrook D, Hackshaw KV, Edwards C. Laboratory pain perception and clinical pain in post-menopausal women and age-matched men with osteoarthritis: relationship to pain coping and hormonal status. PAIN 2004;112:274-81.

[20] Grotle M, Brox JI, Veierod MB, Glomsrod B, Lonn JH, Vollestad NK. Clinical course and prognostic factors in acute low back pain: patients consulting primary care for the first time. Spine (Phila Pa 1976) 2005;30:976-82.

[21] Hancock MJ, Maher CG, Latimer J, Herbert RD, McAuley JH. Can rate of recovery be predicted in patients with acute low back pain? Development of a clinical prediction rule. Eur J Pain 2009;13:51-5.

[22] Hasenbring MI, Hallner D, Klasen B, Streitlein-Bohme I, Willburger R, Rusche $\mathrm{H}$. Pain-related avoidance versus endurance in primary care patients with subacute back pain: psychological characteristics and outcome at a 6-month follow-up. PAIN 2012;153:211-17.

[23] Heneweer H, Aufdemkampe G, van Tulder MW, Kiers H, Stappaerts KH, Vanhees L. Psychosocial variables in patients with (sub)acute low back pain: an inception cohort in primary care physical therapy in The Netherlands. Spine (Phila Pa 1976) 2007;32:586-92.

[24] Henschke N, Maher CG, Refshauge KM, Herbert RD, Cumming RG, Bleasel J, York J, Das A, McAuley JH. Prognosis in patients with recent onset low back pain in Australian primary care: inception cohort study. BMJ 2008;337:a171.

[25] Hestbaek L, Leboeuf-Yde C, Manniche C. Low back pain: what is the long-term course? A review of studies of general patient populations. Eur Spine J 2003;12:149-65.

[26] Hoheisel U, Koch K, Mense S. Functional reorganization in the rat dorsal horn during an experimental myositis. PAIN 1994;59:111-18.

[27] Jensen MB, Manresa JB, Andersen OK. A new objective method for acquisition and quantification of reflex receptive fields. PAIN 2015;156: 555-64.

[28] Kovacs FM, Seco J, Royuela A, Corcoll Reixach J, Abraira V. Predicting the evolution of low back pain patients in routine clinical practice: results from a registry within the Spanish National Health Service. Spine J 2012; 12:1008-20.

[29] Lachs MS, Nachamkin I, Edelstein PH, Goldman J, Feinstein AR, Schwartz JS. Spectrum bias in the evaluation of diagnostic tests: lessons from the rapid dipstick test for urinary tract infection. Ann Intern Med 1992;117:135-40.

[30] Laux L, Glanzmann P, Schaffner P, Spielberger CD. State-TraitAngstinventar (STAl). Göttingen: Hogrefe Verlag, 1981.

[31] Linton SJ. A review of psychological risk factors in back and neck pain. Spine (Phila Pa 1976) 2000;25:1148-56.

[32] Maniadakis N, Gray A. The economic burden of back pain in the UK. PAIN 2000;84:95-103.

[33] McMahon SB, Wall PD. Receptive fields of rat lamina 1 projection cells move to incorporate a nearby region of injury. PAIN 1984;19:235-47.

[34] Morley S, Williams AC, Black S. A confirmatory factor analysis of the Beck Depression Inventory in chronic pain. PAIN 2002;99:289.

[35] Neziri AY, Andersen OK, Petersen-Felix S, Radanov B, Dickenson AH, Scaramozzino P, Arendt-Nielsen L, Curatolo M. The nociceptive withdrawal reflex: normative values of thresholds and reflex receptive fields. Eur J Pain 2010;14:134-41.

[36] Neziri AY, Curatolo M, Bergadano A, Petersen-Felix S, Dickenson AH, Arendt-Nielsen L, Andersen OK. New method for quantification and statistical analysis of nociceptive reflex receptive fields in humans. J Neurosci Methods 2009;178:24-30.

[37] Neziri AY, Curatolo M, Limacher A, Nuesch E, Radanov B, Andersen OK, Arendt-Nielsen L, Juni P. Ranking of parameters of pain hypersensitivity according to their discriminative ability in chronic low back pain. PAIN 2012;153:2083-91.

[38] Neziri AY, Haesler S, Petersen-Felix S, Muller M, Arendt-Nielsen L, Manresa JB, Andersen OK, Curatolo M. Generalized expansion of nociceptive reflex receptive fields in chronic pain patients. PAIN 2010; 151:798-805.

[39] O'Neill S, Manniche C, Graven-Nielsen T, Arendt-Nielsen L. Generalized deep-tissue hyperalgesia in patients with chronic low-back pain. Eur J Pain 2007;11:415-20.

[40] Pincus T, Burton AK, Vogel S, Field AP. A systematic review of psychological factors as predictors of chronicity/disability in prospective cohorts of low back pain. Spine (Phila Pa 1976) 2002;27:E109-120.

[41] Puta C, Schulz B, Schoeler S, Magerl W, Gabriel B, Gabriel HH, Miltner WH, Weiss T. Somatosensory abnormalities for painful and innocuous stimuli at the back and at a site distinct from the region of pain in chronic back pain patients. PLoS One 2013;8:e58885.

[42] Rhudy JL, France CR, Bartley EJ, Williams AE, McCabe KM, Russell JL. Does pain catastrophizing moderate the relationship between spinal nociceptive processes and pain sensitivity? J Pain 2009;10:860-9.

[43] Rhudy JL, Martin SL, Terry EL, France CR, Bartley EJ, DelVentura JL, Kerr $\mathrm{KL}$. Pain catastrophizing is related to temporal summation of pain but not temporal summation of the nociceptive flexion reflex. PAIN 2011;152: 794-801.

[44] Rutjes AW, Reitsma JB, Vandenbroucke JP, Glas AS, Bossuyt PM. Casecontrol and two-gate designs in diagnostic accuracy studies. Clin Chem 2005;51:1335-41.

[45] Schouenborg J, Kalliomaki J. Functional organization of the nociceptive withdrawal reflexes. I. Activation of hindlimb muscles in the rat. Exp Brain Res 1990;83:67-78.

[46] Schouenborg J, Weng HR, Kalliomaki J, Holmberg H. A survey of spinal dorsal horn neurones encoding the spatial organization of withdrawal reflexes in the rat. Exp Brain Res 1995;106:19-27.

[47] Shaw WS, Means-Christensen A, Slater MA, Patterson TL, Webster JS, Atkinson $\mathrm{JH}$. Shared and independent associations of psychosocial factors on work status among men with subacute low back pain. Clin J Pain 2007;23:409-16.

[48] Sieben JM, Vlaeyen JW, Portegijs PJ, Verbunt JA, van Riet-Rutgers S, Kester AD, Von Korff M, Arntz A, Knottnerus JA. A longitudinal study on the predictive validity of the fear-avoidance model in low back pain. PAIN 2005;117:162-70.

[49] Sonnenborg FA, Andersen OK, Arendt-Nielsen L. Modular organization of excitatory and inhibitory reflex receptive fields elicited by electrical stimulation of the foot sole in man. Clin Neurophysiol 2000;111:2160-9.

[50] Sterling M. Differential development of sensory hypersensitivity and a measure of spinal cord hyperexcitability following whiplash injury. PAIN 2010;150:501-6.

[51] Swinkels-Meewisse IE, Roelofs J, Schouten EG, Verbeek AL, Oostendorp RA, Vlaeyen JW. Fear of movement/(re)injury predicting chronic disabling low back pain: a prospective inception cohort study. Spine (Phila Pa 1976) 2006;31:658-64.

[52] Terry EL, Thompson KA, Rhudy JL. Experimental reduction of pain catastrophizing modulates pain report but not spinal nociception as verified by mediation analyses. PAIN 2015;156:1477-88.

[53] Wieser S, Horisberger B, Schmidhauser S, Eisenring C, Brugger U, Ruckstuhl A, Dietrich J, Mannion AF, Elfering A, Tamcan O, Muller U. Cost of low back pain in Switzerland in 2005. Eur J Health Econ 2011;12:455-67.

[54] Woolf CJ. Central sensitization: implications for the diagnosis and treatment of pain. PAIN 2011;152(3 suppl):S2-15.

[55] Woolf CJ, Salter MW. Neuronal plasticity: increasing the gain in pain. Science 2000;288:1765-9.

[56] World Medical Association (WMA). WMA declaration of Helsinki-ethical principles for medical research involving human subjects. 59th WMA General Assembly, Seoul, Republic of Korea, October 2008. 